\title{
TUBERCULOSIS OF BRAIN :
}

\section{PRESENTING WITH RECURRENT STROKE}

AND INTRACRANIAL HAEMORRHAGE

(A Case Report)

\author{
Lt Col BN PANDA*, Lt Col RS SHAH+, \\ Maj RRK NAIR*
}

MJAF1 1995, 51 : 139-141

KEY WORDS : Tuberculosis intracranial; Cerebral haemorrhage.

\section{Introduction}

0 troke in the young (less than 45 years of age) is more often observed in India and accounts for $15-30 \%$ of all strokes as compared to $3 \%$ in Western reports [1]. However, transient reversible ischaemic events of abrupt onset or stuttering progression, so typical of cerebrovascular accidents, are not seen in case of stroke-like presentation in CNS tuberculosis $[2,3]$. Though panarteritic changes and necrotizing lesions have been described earlier, haemorrhagic strokes are considered exceedingly rare [1-4]. We report a young male patient who suffered 2 strokes during a period of $\mathbf{2 0}$ months. The first resulted in right-8ided hemiplegie which totally recovered, but the second episode resulted in death. Autopsy showed bilateral intracerebral heemorrhage.

\section{CASE REPORT}

B], a male aged 30 years, who belonged to $\mathrm{Ma}$ harashtra developed an episode of tonic-clonic selzure associated with loss of consciousness and bladder incontinence. He was brought to hospital within 2 hours of onset of symptoms and was found to be comotose. Response to pain was elicited from only the left balf of the body. His temperature was $102^{\circ} \mathrm{F}$ (rectal), pulse 114/minute and blood pressure 170/94 $\mathrm{mm}$ of $\mathrm{Hg}$. There was external rotation of the right lower limb which displayed brisk deep tendon jerks and extensor plentar response.

"Classified Spectalist (Medicine and Chest Disease), Military Hospital (CTC), Pune 411 040. + Classified Specialiat (Pathology), Military Hospital, Ahmedabad; \#Classified Specialist (Pathology), 150 Base Hospital, C/O 56 APO. 
140 PANDA, SHAH and NAIR

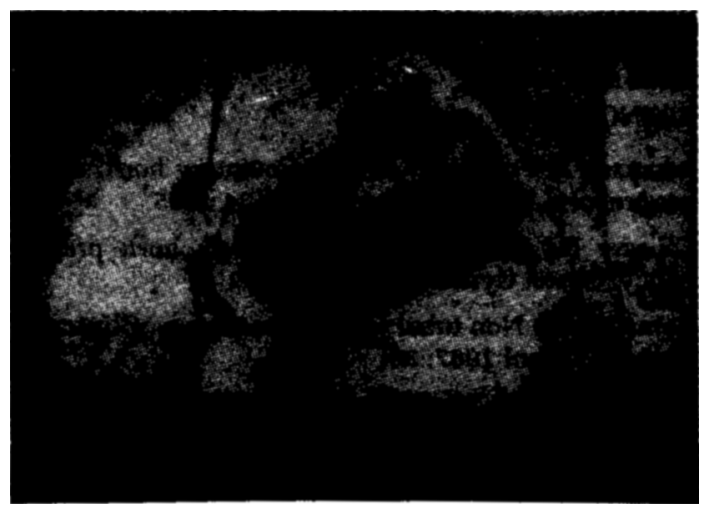

Fig. 1 : Sagittal secion of brain showing blood clots in the lateral ventricle.(Lt).

Right sided facial asymmetry was also noted. Pupillary size and reactions were normal. There was no other cranial nerve deficit. Examination of fundus did not show any papilloedema. Examination of the cardiovascular and respiratory systems were non-contributory. Review of his old medical documents showed that he had an earlier episode of right sided hemiplegia with VII nerve palsy 20 months ago. Blood biochemical parameters, electrocardiogram, cerebrospinal fluid picture and radiography of the chest were then normal. He recovered in two weeks. After 6 weeks of convalescence he was reviewed at a neurology centre where clinical evaluation and computerised tomographic (CT) scan of brain were reported to be normal. He was labelled as a case of occlusive stroke involving left middle cerebral artery territory. Subsequently he was reviewed by us twice at 6 monthly intervals and no sequelae of his neurological event could be demonstrated. He did not have any past or family history of diabetes mellitus, hypertension or tuberculosis. The present episode of convulsion and stroke was not preceded by any febrile episode, headache, vomiting or aura. Initial investigation on admission revealed blood ESR $18 \mathrm{~mm}$ fall in first hour. $\mathrm{Hb}$ was 15,0 g/dL, TLC - 16,400/cumm, DLC - polymorphonuclear leucocytes - $92 \%$, lymphocytes - $6 \%$, monocytes - $1 \%$ and eosinophils $* 1 \%$. Malarial parasite was not detected in peripheral blood smear. Urine analysis showed a normal profile. Blood urea, random blood sugar and total serum bilirubin were normal. Total serum proteins, albumin, globulin and transaminase levels were also within normal limits. Radiography of the chest was normal. CT scan of brain could not be done as the facilities were not available.

CSF done at admission revealed that pressure was normal. It was slightly turbid with $\mathrm{RBC} 950 / \mathrm{mm}^{3}$, WBC $20 / \mathrm{mm}^{3}$, predominant cell lymphocyte. Proteins were 90 $\mathrm{mg} / \mathrm{dL}$ with increased globulins. Sugar was $82 \mathrm{mg} / \mathrm{dL}$. Gram as well as Ziehl-Neelsen stain of CSF did not show any bacteria.

A 12-lead electrocardiogram showed sinus tachycardia. After admission he continued to be febrile. He was presumptively treated with antitubercular drugs,
MJAFI, $51: 2$, APRIL 1995

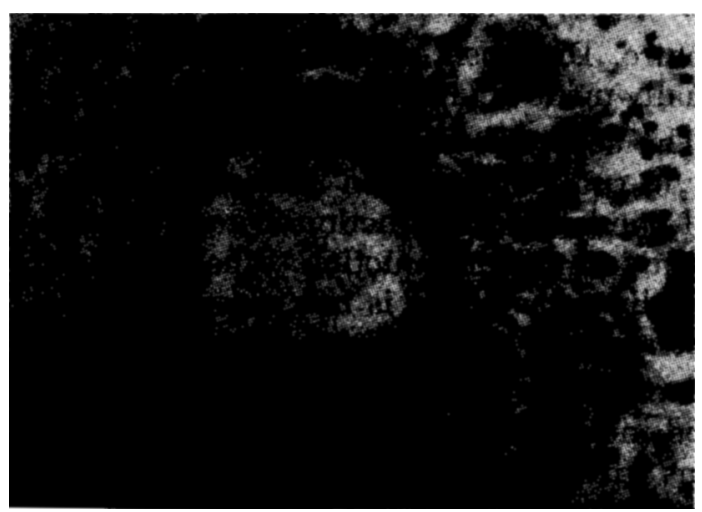

Fig. 2 : Microscopic section of 'mid brain' showing epitheloid granuloma with gaint cells ( $x$ 200).

glucocorticoids and intravenous quinine. On the second day of admission, his coma deepened, plantars became extensor bilaterally and fever persisted. On third day of admission, response to painful stimuli was diminished. He died less than 60 hours after onset of symptoms. Repeat CSF study done immediately after death showed CSF to be uniformly mixed with blood.

Post-mortem examination showed all viscera including heart and lungs to be normal. There was no evidence of significant atherosclerosis in the aorta, carotids or other major vessels. The brain and meninges weighed $1400 \mathrm{~g}$. Left cerabral hemisphere in the temporal region showed a bulge with softening. On cut section, both the cerebral hemispheres showed massive intracerebral bleeding with clots being more marked on the left side. Large areas of left temporal region were replaced by blood clots. Blood clots were also present in both lateral ventricules (Fig 1).

Cerebellum also showed areas of haemorrhage with clots. Lymph node and spleen were normal. Adrenals were congested.

Microscopic examination of the brain showed smalland medium-sized vessels, especially leptomeningeal ones, with aneurysmal dilatations, many showing int mal roughening and medial fragmentation. Leptomeninges showed mononuclear infiltration. Section examined from brain stem (pons, midbrain and medulla) showed areas of haemorrhage, microscopic granuloma, evidence of necrosis, and infiltration with round cells, epitheloid cells and fair number of multinucleated giant cells. A few of them were Langhan's type (Fig 2). ZiehlNeelsen stain of the tissue did not show any acid-fast bacillus in brain or any other organ.

\section{Discussion}

Neurotuberculosis is always caused by secondary dissemination from a primary focus elsewhere in the body (3-5). In the CNS, tuberculosis can affect either the meninges or the brain parenchyma. CNS involvement 
seems to be as high as $45 \%$ in paediatric patients with tuberculosis and it also accounts for $21 \%$ of all intracranial space occupying lesions as reported by Dastur and Lalitha [5]. Fortunately the incidence also seems to be declining [5]. However, tuberculosis as a cause of stroke is a rarity as mentioned in most of the Western textbooks [10]. As far as focal neurological deficit is concerned, hemiparesis was detected in $19 \%$ of cases of tubercular meningitis (TBM) by Thomas et al [7]. The pathogenesis of this vasculopathy in TBM is considered to be the periadventitial inflammation and proliferative subintimal fibrotic lesions consequent to chronic inflammatory processes $[7,8]$. Possibly there also exists strangulatory effect of besal exudates which produce ischaemic softening of cerebral tissue causing sensory motor deficit $[3,5]$. It is more common to find involvement of middle cerebral territory then vertebrobasilar system.

It is interesting to note that in most cases of cerebral arteritis and cerebral softening as described by Destur and Lalitha [5], and by Dalal and Dalal [3], there have been some faatures of pulmonary tuberculosis or TBM to start with. We could not find a single report where reversible ischaemic neurological deficit was followed by recurrence of stroke or haemorrhagic event in $1 \frac{1}{2} 2$ to 2 years time, which has happened in our patient. The rapid progression of the second episode, which proved to be fatal, can only be explained by a rapidly progressive immunologic damage in an already sensitised individual. As in TBM, tuberculosis of brain parenchyma also has been attributed to an immunologic process $[3,5,8,9]$. Such antigen - antibody mediated Arthus-type reaction has been postulated in many clinical settings $[3,5]$ as well as in experimental study in monkeys [9]. This case highlights the importance of tuberculosis as a possible cause of stroke-in-young in countries like ours.

\section{REFERENCES}

1. Srinivasan K. Stroke in the young in India - some etiological factors. In : Sinha KK, Chandra P, editors. Progress in clinical neurosciences - 5. Neurological Society of India 1989; 115-32.

2. Special report from World Health Organisation Stroke 1989. Recommendation on stroke prevention, diagnosis and therapy 1407-31.

3. Dalal PM, Dalal KM. Some aspects of neurotuberculosis in India. In : Sinha KK, Chandra P, editors. Progress in clinical neurosciences - 5. Neurological Society of India 1989; 118-32.

4. Goldzieher JW, Lisa JR. Gross cerebrel haemorrhage in tuberculous meningitis and meningoencephalitis. Am J Path 1947; $23: 133-45$.

5. Dastur DK, Lalitha VS. The many facets of neurotuberculosis. An epitome of neuropathology. In : Zimmerman HM, editor. Progress in Neuropathology (2) New York : Grune Straton, 1973; 351-408.

6. Dalal PM. Stroke in the young in west central India. In : Goldstein Met al editors. Advances in Neurology. New York : Raven Press, 1979; 339-48.

7. Thomas MD, Chopra JS, Walia BNS. Tubercular meningitis (TBM). A clinical study of 232 cases. J Assoc Physician India 1977; $25: 633-9$.

8. Shankar SK. Tuberculous vasculopathy - Revisited. In : Sinha KK, Chandra P, editors. Progress in Clinical Neurosciences - 5. Neurological Society of India 1989; 93-101.

9. Tandon PN, Singh B, Mahapatra LN, Mohankumar. Effect of immunisation and sensitisation on tuberculous lesion of the central nervous system in monkeys. In : Kapila CC et al, editors. Tuberculosis of the nervous system. New Delhi : Indian Acad Med Sci $1974 ; 213$.

10. Warlow C. Diseases of cerebral circulation : Causes of stroke. In : Walton J, editor. Brain diseases of nervous system, 10th ed. London : Oxford Univeristy Press, 1994; 226. 